\title{
Producción de plantas de Tabebuia heptaphylla en diferentes sustratos y niveles de irrigación, en condiciones de invernadero
}

\author{
Seedlings production of Tabebuia heptaphylla in different substrates and levels \\ of irrigation, in greenhouse conditions
}

\author{
Débora Zumkeller Sabonaro**, João Antonio Galbiattib, Rinaldo César de Paula ${ }^{\mathrm{b}}$, José Luis Soto Gonzales \\ *Autor de correspondencia: aUniversidad Estadual Paulista, UNESP, Biología Vegetal, Rio Claro, Brasil, tel. 11-72747507, \\ dzsabonaro@hotmail.com \\ bUniversidad Estadual Paulista, UNESP-FCAV, Jaboticabal, San Pablo, Brasil.
}

\begin{abstract}
SUMMARY
The objective of this research was to evaluate the growth of Tabebuia heptaphylla seedlings in distinct substrates with different amounts of urban waste compost and the relation between this growth and irrigation. A completely randomized experimental design was used, with a factorial arrangement of 15 substrates and 2 irrigation levels. The substrates were composed by the combination of different materials: urban waste, tanned cattle manure, vermiculite, soil and the commercial form Plantmax®. For the study of the seedlings development, the following characteristics were evaluated: plant height, stem diameter at soil level, number of leaves, above ground dry matter, root system dry matter, relation between plant height and stem base diameter, Dickson quality index and relation between plant height and above ground dry matter. Evaluations of plant height, stem diameter at soil level and number of leaves were made at 75, 90,105, 120, 135 and 150 days after sowing. According to the results, it was concluded that urban waste compost does not increase plant development. Significant differences in relation to the irrigation levels were found, with better results for the $150 \%$ irrigation level compared to $100 \%$ evapotranspiration.
\end{abstract}

Key words: native forest species, urban solid waste, height of plants.

\section{RESUMEN}

El estudio tuvo como objetivo evaluar el comportamiento de plántulas de Tabebuia heptaphylla producidas en sustratos con incorporación de diferentes cantidades de residuos urbanos y con dos regímenes de riego. El diseño estadístico utilizado consistió en bloques aleatorizados con 30 tratamientos en un esquema factorial de 15 × 2 (15 sustratos y dos niveles de irrigación), con dos repeticiones. Los sustratos fueron constituidos de diferentes combinaciones de: compuesto de residuo urbano, estiércol curtido, vermiculita más suelo, y sustrato comercial Plantmax ${ }^{\circledR}$. Para el análisis de crecimiento de las plántulas se consideró: altura de la parte aérea, diámetro de la base del tallo de la planta, número de hojas, materia seca de la parte aérea y sistema radicular, relación altura de la parte aérea/diámetro de la base del tallo, índice de calidad de Dickson y relación altura de la parte aérea/materia seca de la parte aérea. Las características evaluadas fueron medidas a los 75, 90, 105, 120, 135 y 150 días después de la siembra. Se concluye que el compuesto de residuo urbano no favoreció el crecimiento de las plantas. El crecimiento de las plantas fue superior con el nivel de irrigación de $150 \%$ de la evapotranspiración con respecto al de $100 \%$.

Palabras clave: especies forestal autóctona, compuesto de residuos urbanos, altura de plantas.

\section{INTRODUCCIÓN}

La producción de residuo urbano está relacionada con el crecimiento de la población y la industria en todo el mundo. El aumento de la producción de residuos sólidos preocupa a las autoridades gubernamentales y científicas, principalmente por la falta de lugares seguros para su disposición que eviten riesgos de contaminación ambiental. El Instituto Brasileño de Geografía y Estadística (IBGE 2005) informa que la cantidad media de residuo sólido producida en Brasil es de $0,6 \mathrm{~kg}$ por día, por habitante, variando de $0,4 \mathrm{~kg}$ para la región noreste a 1,1 kg para la región sureste. Especialistas reportan que la generación de residuos domésticos aumentó visiblemente después de la introducción en 1994 del Plan Real. Los residuos sólidos pueden perjudicar la calidad del agua de la napa freática, que corre riesgo de contaminación, y traen perjuicios a la salud pública (San Martin 2000). Es evidente que estrategias de reutilización de residuos contribuyen a la reducción de su potencial dañino y constituyen aspectos fundamentales en proyectos ambientales y en proyectos de políticas públicas. La obtención de productos de valor económico, como resultado de un procesamiento adecuado de estos residuos sólidos, se configuraba hasta hace poco tiempo como objetivo secundario. 
Según Guerrini y Trigueiro (2004), los sustratos para la producción de plántulas son definidos como el medio adecuado para sustentación de plantas y deben presentar propiedades que permitan la retención de cantidades suficientes y necesarias de agua, oxígeno y nutrientes, además de ofrecer $\mathrm{pH}$ compatible con la especie vegetal, ausencia de elementos químicos en niveles tóxicos y conductividad eléctrica adecuada. La fase sólida de sustrato debe ser constituida por una mezcla de partículas minerales y orgánicas. El estudio de la disposición porcentual de estos componentes es importante, ya que ellos pueden ser fuente de nutrientes y actuarán directamente sobre el crecimiento y desarrollo de las plantas. Por lo tanto, en concordancia con la disposición cuantitativa y cualitativa de los materiales minerales y orgánicos empleados, las plántulas serán influenciadas por carencia de nutrientes y por disponibilidad de agua y oxígeno. Para Schmitz et al. (2002) el uso de los residuos como componentes de sustratos propicia la obtención de materiales alternativos de fácil disponibilidad y de bajo costo, auxiliando en la minimización de la contaminación recurrente de la acumulación de residuos en el ambiente.

La producción de plántulas de uso forestal de buena calidad incluye los procesos de germinación de semillas, crecimiento y formación del sistema radicular y parte aérea, que están directamente relacionados con características que definen el nivel de eficiencia de los sustratos, tales como: aireación, drenaje, retención de agua y disponibilidad balanceada de nutrientes. A su vez, las características de los sustratos están altamente correlacionadas entre sí: macroporos con aireación y drenaje, y microporos con la retención de agua y nutrientes (Gonçalves y Poggiani 1996, Caldeira et al. 2000).

Tabebuia heptaphylla (Vellozo) Toledo pertenece a la familia Bignonaceae. Es una especie de alto valor económico, principalmente por las propiedades de su madera y de sus extractos foliares (Ettori et al. 1996). Posee crecimiento moderado (Coelba 2002), sin embargo, comparada con otras especies forestales nativas, $T$. heptaphylla tiene crecimiento relativamente rápido, sirviendo muy bien a la reforestación (Longhi 1995).

Debido a la preocupación ambiental relativa al depósito de residuos sólidos, la utilización de materiales de desecho de origen vegetal y animal para la producción de plántulas de especies forestales nativas es de importancia y el éxito en la recuperación de áreas degradadas vía reforestación depende en gran parte de la calidad de plántulas producidas.

En atención a lo anterior y con la intención de dar un direccionamiento a residuos urbanos para su uso en la produccion de plantas forestales, el presente trabajo tiene por objetivo evaluar el comportamiento de T. heptaphylla en diferentes sustratos artificiales y dos niveles de irrigación, utilizándose diferentes cantidades de compuestos de residuo urbano.

\section{MÉTODOS}

El experimento fue realizado en invernadero, en el área perteneciente al Departamento de Ingeniería Rural de la Facultad de Ciencias Agrarias y Veterinarias de la UNESP, Campus de Jaboticabal, San Pablo, Brasil ( $21^{\circ} 15^{\prime} 15^{\prime \prime} \mathrm{S}$, $48^{\circ} 18^{\prime} 09^{\prime \prime}$ O y 595 m s.n.m.). La clasificación climática para esta región (Köppen 1973) es de tipo Cwa, subtropical húmedo con estiaje en invierno; la precipitación y las temperaturas medias anuales se sitúan próximas de 1.400 $\mathrm{mm}$ y $21^{\circ} \mathrm{C}$, respectivamente.

Las semillas para la producción de plántulas de T. heptaphylla fueron obtenidas en el Vivero Experimental de Plantas Ornamentales y Forestales de la Facultad de Ciencias Agrarias y Veterinarias, Jaboticabal, San Pablo, Brasil. Fueron utilizados tubetes de plástico rígido $(13 \mathrm{~cm}$ de altura y $160 \mathrm{~cm}^{3}$ de volumen), con diferentes sustratos, bajo sombreamiento de $30 \%$ de capacidad de retención de luz al inicio del experimento. En cada tubete fueron depositadas dos semillas, para posterior raleo a los 30 días. Siete días después del raleo, las plántulas fueron expuestas a pleno sol.

Los ensayos constaron de 15 sustratos, resultantes de la combinación de sustrato comercial Plantmax ${ }^{\circledR}$, ampliamente utilizado en la producción de plantas de especies forestales nativas en Brasil, con estiércol bovino, compost de residuos urbanos, vermiculita $15 \%$ y suelo $10 \%$ (cuadro 1 ).

Cuadro 1. Composición volumétrica (\%) de los sustratos utilizados para producción de plántulas de Tabebuia heptaphylla.

Percentage of materials used on substrates composition for Tabebuia heptaphylla seedlings production.

\begin{tabular}{lccc}
\hline Sustratos & Plantmax $^{\circledR}$ & Estiércol & $\begin{array}{c}\text { Compuesto de } \\
\text { residuos urbanos }\end{array}$ \\
\hline 1 & 75 & - & - \\
2 & 60 & 15 & - \\
3 & 45 & 30 & - \\
4 & 30 & 45 & - \\
5 & 15 & 60 & - \\
6 & - & 75 & - \\
7 & 60 & - & 15 \\
8 & 45 & - & 30 \\
9 & 30 & - & 45 \\
10 & 15 & - & 60 \\
11 & - & - & 75 \\
12 & - & 60 & 15 \\
13 & - & 45 & 30 \\
14 & - & 30 & 45 \\
15 & - & 15 & 60 \\
\hline
\end{tabular}


Los residuos orgánicos para el sustrato fueron obtenidos en San José de Río Preto (SP), ciudad que realiza la colecta selectiva de residuos urbanos y el apropiado compostaje de los residuos orgánicos. La vermiculita utilizada fue de textura media.

Se utilizaron muestras de suelo oriundas de Oxisoles rojos de la Floresta Atlántica, textura media, obtenida a una profundidad de $0-20 \mathrm{~cm}$. Los análisis químicos y granulométricos (cuadro 2) fueron realizados en el Departamento de Suelos y Abonos de la Facultad de Ciencias Agrarias y Veterinarias de Jaboticabal, conforme metodología recomendada por Tomé Júnior (1997).

La incorporación inicial de abono mineral y de cobertura fueron realizadas según recomendaciones de Gonçalves et al. (1997) para establecer dos niveles de irrigación. Inicialmente se estimó la evapotranspiración (ET) según Broner y Law (1991), para definir las láminas de agua a ser aplicadas en la irrigación. Con base en esas medidas fueron definidos dos niveles de irrigación: $100 \%$ y $150 \%$ de la evapotranspiración, respectivamente. Los riegos fueron realizados todos los días y manualmente.

El cultivo en contenedores requiere riegos y fertilización frecuente, por lo tanto es necesario conocer las propiedades químicas y físicas de los sustratos, por ser factores determinantes en el manejo y control de calidad de cultivos y en la producción de plántulas. Conforme al cuadro 3 , el pH fue determinado con un potenciómetro. Los análisis de la densidad aparente fueron realizados según la metodología propuesta por Gonçalves y Poggiani (1996).

La evaluación del crecimiento de plántulas de la especie estudiada fue basada en las variables altura de la parte aérea $(\mathrm{H}$, en $\mathrm{cm})$, diámetro del tallo a la altura de $3 \mathrm{~cm}$ de la superficie del sustrato (D, en $\mathrm{mm}$ ), número de hojas (NH) y materia seca de la parte aérea (MSPA, en g) y del sistema radicular (MSR, en g). Las evaluaciones de
H, D y NH fueron efectuadas a los 75, 90, 105, 120, 135 y 150 días después de la siembra (DDS). Las plántulas fueron evaluadas hasta llegar a condiciones adecuadas para traslado a campo definitivo.

Al final del experimento, para tomar las medidas de MSPA y MSR, las plantas fueron separadas en raíz y parte aérea, lavadas en agua destilada y colocadas a secar en estufa con circulación de aire forzado a $65^{\circ} \mathrm{C}$, hasta masa constante. Con los datos obtenidos también fueron calculados diferentes índices de calidad de plántulas: materia seca total $($ MST $=$ MSPA + MSR $)$, relación altura de la parte aérea y diámetro del tallo (H/D), relación altura de la parte aérea y materia seca de la parte aérea (H/MSPA), relación materia seca de la parte aérea y materia seca de las raíces (MSPA/MSR) e índice de calidad de Dickson (IDC) (Dickson et al. 1960) por la fórmula IDC = MST/ [(H/DC) + (MSPA/MSR $)]$.

Fue utilizado el diseño experimental en bloques aleatorizados, con 30 tratamientos, con un esquema factorial de 15 x 2 (15 sustratos y dos niveles de irrigación) con dos repeticiones (el análisis de varianza fue con $\mathrm{F}$ al $1 \mathrm{y}$ $5 \%$ de probabilidad, siendo las medias comparadas por la prueba de Scott - Knott (Scott y Knott 1974).

\section{RESULTADOS}

Se verificó que los sustratos 2 y 3 proporcionaron mayores valores de altura de la parte aérea y del diámetro de la base del tallo de la planta (cuadro 4); los sustratos 1, 2 y 3 fueron los que proporcionaron los mayores valores de 90 a 150 DDS. En cuanto al número de hojas, considerados todos los períodos evaluados, los sustratos 2 y 3 propiciaron los mayores valores, también; se verificó que los sustratos 2 y 3 proporcionaron mayores valores de altura de la

Cuadro 2. Datos del análisis químico y granulométrico de suelo. Oxisol rojo de la Floresta Atlántica, Brasil.

Chemical and granulate analyses in soil. Oxisol red of the Atlantic, Brazilian.

\begin{tabular}{|c|c|c|c|c|c|c|c|c|c|c|c|c|c|c|}
\hline $\mathrm{pH}$ & M.O. & $\mathrm{P}$ & K & $\mathrm{Ca}$ & $\mathrm{Mg}$ & $\mathrm{H}+\mathrm{Al}$ & SB & $\mathrm{T}$ & $\mathrm{B}$ & Arcilla & Limo & $\begin{array}{c}\text { Arena } \\
\text { fina }\end{array}$ & $\begin{array}{l}\text { Arena } \\
\text { gruesa }\end{array}$ & $\begin{array}{l}\text { Clase } \\
\text { textural }\end{array}$ \\
\hline $\mathrm{CaCl}_{2}$ & $\mathrm{~g} \mathrm{dm}{ }^{-3}$ & $\begin{array}{c}\text { Resina } \\
\mathrm{Mg} \mathrm{m}^{-3}\end{array}$ & ---- & 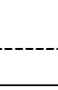 & $\mathrm{mm}$ & $\mathrm{m}^{-3}$ & & & $\%$ & ---- & & -- & --- & \\
\hline 4,2 & 21 & 2 & 0,6 & 5 & 3 & 42 & 8,6 & 50 & 17 & 320 & 40 & 270 & 370 & media \\
\hline
\end{tabular}

M.O.: materia orgánica. SB: suma de bases. T: Capacidad de intercambio catiónico. B: bases saturadas.

Cuadro 3. Características físicas y químicas de los quince tratamientos probados como sustratos después de su formulación. Chemical and physical characteristics in the fifteen tested substrates after their formulation.

\begin{tabular}{|c|c|c|c|c|c|c|c|c|c|c|c|c|c|c|c|}
\hline Sustratos & 1 & 2 & 3 & 4 & 5 & 6 & 7 & 8 & 9 & 10 & 11 & 12 & 13 & 14 & 15 \\
\hline $\mathrm{pH}$ en $\mathrm{CaCl}_{2} 0,01 \mathrm{M}$ & 4,2 & 6,2 & 6,9 & 7,4 & 7,5 & 8,3 & 6,5 & 6,9 & 7,0 & 7,2 & 7,2 & 8,0 & 7,8 & 7,7 & 7,7 \\
\hline Densidad $\left(\mathrm{g} / \mathrm{cm}^{3}\right)$ & 0,50 & 0,47 & 0,50 & 0,51 & 0,55 & 0,52 & 0,55 & 0,58 & 0,76 & 0,77 & 0,77 & 0,56 & 0,65 & 0,58 & 0,65 \\
\hline
\end{tabular}


Cuadro 4. Resumen del análisis de varianza para altura de la parte aérea $(\mathrm{H}, \mathrm{cm})$; diámetro de la base del tallo de la planta (D, mm); número de hojas de plantas de Tabebuia heptaphylla, obtenidas a los 75, 90, 105, 120, 135 y 150 días después de la siembra (DDS) en los diferentes sustratos.

Results of statistical analyses: seedlings height (cm), stem -base diameter in (mm), leaf number of plants Tabebuia heptaphylla, obtained $75,90,105$, 120, 135 and 150 days after sowing (DDS) in the different substrates.

Períodos evaluados (DDS)

\begin{tabular}{|c|c|c|c|c|c|c|c|c|c|c|c|c|c|c|c|c|c|c|}
\hline \multirow{3}{*}{$\begin{array}{l}\text { Sustratos } \\
(\mathrm{S})\end{array}$} & \multirow{2}{*}{\multicolumn{6}{|c|}{ Altura de la parte aérea $(\mathrm{H}, \mathrm{cm})$}} & \multicolumn{6}{|c|}{ Períodos evaluados (DDS) } & & & & & & \\
\hline & & & & & & & \multicolumn{6}{|c|}{ Diámetro de la base del tallo de la planta (D, mm) } & \multicolumn{6}{|c|}{ Número de hojas } \\
\hline & 75 & 90 & 105 & 120 & 135 & 150 & 75 & 90 & 105 & 120 & 135 & 150 & 75 & 90 & 105 & 120 & 135 & 150 \\
\hline 1 & $6,4 \mathrm{~b}$ & $7,0 \mathrm{c}$ & $7,6 \mathrm{c}$ & $9,1 \mathrm{c}$ & $11,1 \mathrm{c}$ & $12,9 \mathrm{c}$ & $1,5 \mathrm{a}$ & $1,8 \mathrm{a}$ & $1,9 \mathrm{a}$ & $2,1 \mathrm{a}$ & $2,1 \mathrm{a}$ & $2,2 \mathrm{a}$ & $9,3 \mathrm{~b}$ & $11,2 \mathrm{~b}$ & $12,0 \mathrm{c}$ & $14,9 \mathrm{c}$ & $16,2 \mathrm{~b}$ & $19,1 \mathrm{~b}$ \\
\hline 2 & $7,8 \mathrm{a}$ & $9,2 \mathrm{a}$ & $12,5 \mathrm{a}$ & $15,0 \mathrm{a}$ & 16,6 a & $18,8 \mathrm{a}$ & $1,4 \mathrm{a}$ & $1,7 \mathrm{a}$ & $1,9 \mathrm{a}$ & $2,1 \mathrm{a}$ & $2,2 \mathrm{a}$ & $2,3 \mathrm{a}$ & $10,2 \mathrm{a}$ & $13,8 \mathrm{a}$ & $17,0 \mathrm{a}$ & 19,9 a & $23,2 \mathrm{a}$ & $24,5 \mathrm{a}$ \\
\hline 3 & $8,6 \mathrm{a}$ & $10,2 \mathrm{a}$ & 13,2 a & $15,3 \mathrm{a}$ & 16,4 a & $17,7 \mathrm{a}$ & $1,5 \mathrm{a}$ & $1,7 \mathrm{a}$ & $2,0 \mathrm{a}$ & $2,1 \mathrm{a}$ & $2,3 \mathrm{a}$ & $2,4 \mathrm{a}$ & $11,7 \mathrm{a}$ & $13,5 \mathrm{a}$ & 19,3 a & $20,7 \mathrm{a}$ & 22,1 a & $22,7 \mathrm{a}$ \\
\hline 4 & $6,5 \mathrm{~b}$ & $8,0 \mathrm{~b}$ & $11,3 \mathrm{~b}$ & $12,7 \mathrm{~b}$ & $13,7 \mathrm{~b}$ & $14,7 \mathrm{~b}$ & $1,3 \mathrm{~b}$ & $1,4 \mathrm{~b}$ & $1,6 \mathrm{~b}$ & $1,7 \mathrm{~b}$ & $1,8 \mathrm{~b}$ & $1,9 \mathrm{~b}$ & $10,5 \mathrm{a}$ & $13,6 \mathrm{a}$ & $15,2 \mathrm{~b}$ & $17,8 \mathrm{~b}$ & $21,6 \mathrm{a}$ & $22,0 \mathrm{a}$ \\
\hline 5 & $5,9 \mathrm{c}$ & $7,2 \mathrm{c}$ & $9,7 \mathrm{~b}$ & $11,8 \mathrm{~b}$ & $11,7 \mathrm{~b}$ & $13,8 \mathrm{~b}$ & $1,2 \mathrm{~b}$ & $1,4 \mathrm{~b}$ & $1,6 \mathrm{~b}$ & $1,7 \mathrm{~b}$ & $1,8 \mathrm{~b}$ & $2,0 \mathrm{~b}$ & $9,0 \mathrm{~b}$ & $12,0 \mathrm{a}$ & $13,8 \mathrm{~b}$ & $16,0 \mathrm{~b}$ & $18,0 \mathrm{~b}$ & $18,5 \mathrm{~b}$ \\
\hline 6 & $5,1 \mathrm{c}$ & $6,2 \mathrm{c}$ & $9,7 \mathrm{~b}$ & $10,2 \mathrm{c}$ & $13,0 \mathrm{~b}$ & $12,6 \mathrm{c}$ & $1,1 \mathrm{~b}$ & $1,3 \mathrm{~b}$ & $1,5 \mathrm{~b}$ & $1,6 \mathrm{~b}$ & $1,7 \mathrm{~b}$ & $2,0 \mathrm{~b}$ & $9,4 \mathrm{~b}$ & $11,8 \mathrm{a}$ & $15,3 \mathrm{~b}$ & $16,7 \mathrm{~b}$ & $17,1 \mathrm{~b}$ & $17,9 \mathrm{~b}$ \\
\hline 7 & $4,4 \mathrm{c}$ & $5,2 \mathrm{c}$ & $5,9 \mathrm{c}$ & $7,6 \mathrm{~d}$ & $12,0 \mathrm{c}$ & $12,0 \mathrm{c}$ & $1,3 \mathrm{a}$ & $1,4 \mathrm{~b}$ & $1,6 \mathrm{~b}$ & $1,7 \mathrm{~b}$ & $1,8 \mathrm{~b}$ & $1,8 \mathrm{~b}$ & $6,8 \mathrm{c}$ & 8,4 a & $9,7 \mathrm{c}$ & $13,9 \mathrm{c}$ & $17,2 \mathrm{~b}$ & $18,2 \mathrm{~b}$ \\
\hline 8 & $4,6 \mathrm{c}$ & $4,9 \mathrm{~d}$ & $5,9 \mathrm{c}$ & $6,5 \mathrm{~d}$ & $9,5 \mathrm{~d}$ & $9,9 \mathrm{~d}$ & $1,3 \mathrm{a}$ & $1,5 \mathrm{~b}$ & $1,7 \mathrm{~b}$ & $1,8 \mathrm{~b}$ & $1,9 \mathrm{~b}$ & $1,9 \mathrm{~b}$ & $7,7 \mathrm{c}$ & $9,4 \mathrm{~b}$ & $11,0 \mathrm{c}$ & $14,7 \mathrm{c}$ & $17,7 \mathrm{~b}$ & $20,9 \mathrm{a}$ \\
\hline 9 & $4,6 \mathrm{c}$ & $5,0 \mathrm{~d}$ & $6,0 \mathrm{c}$ & $6,6 \mathrm{~d}$ & $9,2 \mathrm{~d}$ & $9,4 \mathrm{~d}$ & $1,2 \mathrm{~b}$ & $1,5 \mathrm{~b}$ & $1,6 \mathrm{~b}$ & $1,7 \mathrm{~b}$ & $1,8 \mathrm{~b}$ & $1,9 \mathrm{~b}$ & $5,7 \mathrm{c}$ & $7,8 \mathrm{~b}$ & $9,8 \mathrm{c}$ & $11,6 \mathrm{c}$ & $16,4 \mathrm{~b}$ & $17,2 \mathrm{~b}$ \\
\hline 10 & $5,2 \mathrm{c}$ & $5,9 \mathrm{~d}$ & $6,9 \mathrm{c}$ & $7,7 \mathrm{~d}$ & $10,6 \mathrm{c}$ & $11,5 \mathrm{c}$ & $1,2 \mathrm{~b}$ & $1,5 \mathrm{~b}$ & $1,7 \mathrm{~b}$ & $1,8 \mathrm{~b}$ & $1,8 \mathrm{~b}$ & $1,9 \mathrm{~b}$ & $7,0 \mathrm{c}$ & $8,6 \mathrm{~b}$ & $12,0 \mathrm{c}$ & $16,8 \mathrm{c}$ & $16,9 \mathrm{~b}$ & $19,0 \mathrm{~b}$ \\
\hline 11 & $5,2 \mathrm{c}$ & $6,1 \mathrm{c}$ & $6,8 \mathrm{c}$ & $7,6 \mathrm{~d}$ & $8,4 \mathrm{~d}$ & $9,1 \mathrm{~d}$ & $1,1 \mathrm{~b}$ & $1,3 \mathrm{~b}$ & $1,5 \mathrm{~b}$ & $1,6 \mathrm{~b}$ & $1,7 \mathrm{~b}$ & $1,9 \mathrm{~b}$ & $5,8 \mathrm{c}$ & $8,3 \mathrm{~b}$ & $9,4 \mathrm{c}$ & $10,6 \mathrm{c}$ & $12,1 \mathrm{c}$ & $13,4 \mathrm{~b}$ \\
\hline 12 & $5,4 \mathrm{c}$ & $6,6 \mathrm{c}$ & $10,0 \mathrm{~b}$ & $11,3 \mathrm{~b}$ & $12,1 \mathrm{c}$ & $13,5 \mathrm{~b}$ & $1,1 \mathrm{~b}$ & $1,3 \mathrm{~b}$ & $1,5 \mathrm{~b}$ & $1,6 \mathrm{~b}$ & $1,7 \mathrm{~b}$ & $1,8 \mathrm{~b}$ & $7,2 \mathrm{c}$ & $10,6 \mathrm{~b}$ & $14,1 \mathrm{~b}$ & $16,4 \mathrm{~b}$ & $18,2 \mathrm{~b}$ & $19,7 \mathrm{~b}$ \\
\hline 13 & $5,8 \mathrm{c}$ & $6,6 \mathrm{c}$ & $9,7 \mathrm{~b}$ & $10,2 \mathrm{c}$ & $11,4 \mathrm{c}$ & $11,9 \mathrm{c}$ & $1,2 \mathrm{~b}$ & $1,4 \mathrm{~b}$ & $1,6 \mathrm{~b}$ & $1,7 \mathrm{~b}$ & $1,7 \mathrm{~b}$ & $1,8 \mathrm{~b}$ & $8,9 \mathrm{~b}$ & $11,8 \mathrm{a}$ & $15,9 \mathrm{~b}$ & $17,3 \mathrm{~b}$ & $19,1 \mathrm{~b}$ & $19,7 \mathrm{~b}$ \\
\hline 14 & $6,6 \mathrm{~b}$ & $7,2 \mathrm{~d}$ & $10,7 \mathrm{~b}$ & $11,7 \mathrm{~b}$ & $12,6 \mathrm{~b}$ & $14,6 \mathrm{~b}$ & $1,3 \mathrm{~b}$ & $1,5 \mathrm{~b}$ & $1,8 \mathrm{~b}$ & $1,8 \mathrm{~b}$ & $1,9 \mathrm{~b}$ & $1,9 \mathrm{~b}$ & $8,7 \mathrm{~b}$ & $12,3 \mathrm{a}$ & $15,6 \mathrm{~b}$ & $17,0 \mathrm{~b}$ & $18,8 \mathrm{~b}$ & $21,3 \mathrm{a}$ \\
\hline 15 & $4,9 \mathrm{c}$ & $5,7 \mathrm{~d}$ & $7,3 \mathrm{c}$ & $7,9 \mathrm{~d}$ & $9,3 \mathrm{~d}$ & $10,3 \mathrm{~d}$ & $1,2 \mathrm{~b}$ & $1,5 \mathrm{~b}$ & $1,6 \mathrm{~b}$ & $1,7 \mathrm{~b}$ & $1,7 \mathrm{~b}$ & $1,8 \mathrm{~b}$ & $8,3 \mathrm{~b}$ & $9,7 \mathrm{~b}$ & $12,2 \mathrm{c}$ & $13,4 \mathrm{c}$ & $16,7 \mathrm{~b}$ & $18,0 \mathrm{~b}$ \\
\hline Valor $\mathrm{F}$ & $8,17 * *$ & $16,54 * *$ & $17,21 * *$ & $16,02 * *$ & $1229 * *$ & $11,09 * *$ & $3,64 * *$ & $3,35^{*}$ & $3,95 * *$ & $4,30 * *$ & $4,84 * *$ & $5,30 * *$ & $6,19^{* *}$ & $7,84 * *$ & $9,39 * *$ & $6,20 * *$ & $322 *$ & $3,48^{* *}$ \\
\hline
\end{tabular}

Niveles de

irrigación (I)

Períodos evaluados (DDS)

\begin{tabular}{|c|c|c|c|c|c|c|c|c|c|c|c|c|c|c|c|c|c|c|}
\hline $100 \%$ & $5,6 \mathrm{a}$ & $6,7 \mathrm{a}$ & 8,9 a & $9,9 \mathrm{a}$ & $11,1 \mathrm{~b}$ & $12,0 \mathrm{~b}$ & $1,2 \mathrm{~b}$ & $1,4 \mathrm{a}$ & $1,6 \mathrm{a}$ & $1,7 \mathrm{~b}$ & $1,8 \mathrm{a}$ & $1,9 \mathrm{a}$ & $8,2 \mathrm{a}$ & $10,6 \mathrm{a}$ & $13,5 \mathrm{a}$ & $15,1 \mathrm{a}$ & $17,2 \mathrm{~b}$ & $18,4 \mathrm{~b}$ \\
\hline $150 \%$ & $6,0 \mathrm{a}$ & $6,8 \mathrm{a}$ & $8,8 \mathrm{a}$ & $10,2 \mathrm{a}$ & $12,1 \mathrm{a}$ & $13,5 \mathrm{a}$ & $1,3 \mathrm{a}$ & $1,4 \mathrm{a}$ & $1,7 \mathrm{a}$ & $1,8 \mathrm{a}$ & $1,9 \mathrm{a}$ & $2,0 \mathrm{a}$ & $8,6 \mathrm{a}$ & $11,1 \mathrm{a}$ & $13,4 \mathrm{a}$ & $16,0 \mathrm{a}$ & $18,9 \mathrm{a}$ & $20,6 \mathrm{a}$ \\
\hline Valor F & 3,78 NS & $0,49 \mathrm{NS}$ & $0,24 \mathrm{NS}$ & $0,93 \mathrm{NS}$ & $7,32 *$ & $10,72 * *$ & $13,11^{*}$ & $1,24 \mathrm{NS}$ & 2,29 NS & $4,55^{*}$ & 3,20 NS & $0,43 \mathrm{NS}$ & 1,49 NS & 2,47 NS & $0,01 \mathrm{NS}$ & 2,27 NS & $7,02 *$ & $9,16^{* *}$ \\
\hline $\mathrm{S} \times \mathrm{I}$ & $1,13 \mathrm{NS}$ & $3,95 * *$ & $0,75 \mathrm{NS}$ & $0,58 \mathrm{NS}$ & $0,73 \mathrm{NS}$ & $1,02 \mathrm{NS}$ & $1,29 \mathrm{NS}$ & $0,76 \mathrm{NS}$ & $0,85 \mathrm{NS}$ & $0,69 \mathrm{NS}$ & $0,97 \mathrm{NS}$ & $1,02 \mathrm{NS} 2$ & $273 * *$ & $1,49 \mathrm{NS}$ & $0,61 \mathrm{NS}$ & $0,62 \mathrm{NS}$ & $1,19 \mathrm{NS}$ & $1,28 \mathrm{NS}$ \\
\hline $\mathrm{CV} \%$ & 14,5 & 11,0 & 13,4 & 14,2 & 12,7 & 13,4 & 10,3 & 11,0 & 9,8 & 9,5 & 9,4 & 8,4 & 16,2 & 13,5 & 14,2 & 14,8 & 13,5 & 14,5 \\
\hline
\end{tabular}

Medias seguidas por la misma letra, en la columna no difieren entre sí al $5 \%$ de probabilidad por la prueba de Scott y Knott. ns no significativo $(P>0,05)$. $P<0,05 . * * P<0,01$. CV: coeficiente de variación. S x I: interacción entre sustrato e irrigación. 
Cuadro 5. Desdoblamiento de la interacción sustrato-irrigación para el diámetro de la parte aérea/diámetro de la base del tallo y materia seca de la parte aérea/materia seca de la raíz, altura de la parte aérea a los 90 DDS y número de hojas a los 75 DDS, altura de la parte aérea/diámetro de la base del tallo y materia seca de la parte aérea/materia seca de la raíz para la especie Tabebuia heptaphylla.

Deployment of the interaction between substrate-irrigation for the characteristic height of the aerial part $(\mathrm{cm})$ to the 90 D.D.S and number of the 75 leaves to D.D.S. Relation between plant height/stem-base diameter, total dry matter produce, and the relation between plant height/ stem diameter for the species Tabebuia heptaphylla.

\begin{tabular}{|c|c|c|c|c|c|c|c|c|}
\hline \multirow{3}{*}{ Sustratos } & \multirow{2}{*}{\multicolumn{2}{|c|}{$\begin{array}{c}\begin{array}{c}\text { Altura de la parte } \\
\text { aérea }\end{array} \\
90 \text { DDS }(\mathrm{cm})\end{array}$}} & \multirow{2}{*}{\multicolumn{2}{|c|}{$\begin{array}{c}\text { Número de hojas } \\
75 \text { DDS }\end{array}$}} & \multirow{2}{*}{\multicolumn{2}{|c|}{$\begin{array}{c}\begin{array}{c}\text { Diámetro de } \\
\text { la parte aérea }\end{array} \\
\text { H/D }\end{array}$}} & \multirow{2}{*}{\multicolumn{2}{|c|}{$\begin{array}{c}\begin{array}{c}\text { Diámetro de la base } \\
\text { del tallo }\end{array} \\
\text { MSPA/MSR } \\
\end{array}$}} \\
\hline & & & & & & & & \\
\hline & I 1 & I 2 & I 1 & I 2 & I 1 & I 2 & I 1 & I 2 \\
\hline 1 & $7,6 \mathrm{cA}$ & $6,4 \mathrm{bA}$ & $9,3 \mathrm{aA}$ & $9,3 \mathrm{aA}$ & $4,5 \mathrm{cA}$ & $7,4 \mathrm{aB}$ & $1,41 \mathrm{bA}$ & $0,88 \mathrm{bA}$ \\
\hline 2 & $10,0 \mathrm{bA}$ & $8,4 \mathrm{aB}$ & $9,9 \mathrm{aA}$ & $9,3 \mathrm{aA}$ & $8,0 \mathrm{aA}$ & $8,1 \mathrm{aA}$ & $1,26 \mathrm{bA}$ & $1,08 \mathrm{bA}$ \\
\hline 3 & $11,7 \mathrm{aA}$ & $8,8 \mathrm{aB}$ & $12,8 \mathrm{aA}$ & $10,6 \mathrm{aA}$ & $7,0 \mathrm{bA}$ & $8,1 \mathrm{aA}$ & $1,01 \mathrm{bA}$ & $0,95 \mathrm{bA}$ \\
\hline 4 & $7,9 \mathrm{cA}$ & $8,2 \mathrm{aA}$ & $9,1 \mathrm{aA}$ & $11,8 \mathrm{aA}$ & $8,4 \mathrm{aA}$ & $7,2 \mathrm{aA}$ & $2,66 \mathrm{aA}$ & $1,61 \mathrm{aA}$ \\
\hline 5 & $7,4 \mathrm{cA}$ & $7,1 \mathrm{aA}$ & $10,4 \mathrm{aA}$ & $7,5 \mathrm{bB}$ & $6,8 \mathrm{bA}$ & $7,2 \mathrm{aA}$ & $1,66 \mathrm{aA}$ & $2,18 \mathrm{aB}$ \\
\hline 6 & $6,1 \mathrm{dA}$ & $6,2 \mathrm{bA}$ & $10,7 \mathrm{aA}$ & $8,1 \mathrm{bA}$ & $6,5 \mathrm{bA}$ & $7,0 \mathrm{aA}$ & $1,25 \mathrm{bA}$ & $1,72 \mathrm{aA}$ \\
\hline 7 & $6,0 \mathrm{dA}$ & $4,4 \mathrm{cB}$ & $8,0 \mathrm{aA}$ & $5,6 \mathrm{bA}$ & $6,5 \mathrm{bA}$ & $5,9 \mathrm{bA}$ & $0,70 \mathrm{bA}$ & $1,04 \mathrm{bA}$ \\
\hline 8 & $5,0 \mathrm{dA}$ & $4,9 \mathrm{cA}$ & $6,6 \mathrm{bA}$ & $8,7 \mathrm{aA}$ & $4,7 \mathrm{cA}$ & $5,5 \mathrm{bA}$ & $0,46 \mathrm{bA}$ & $0,94 \mathrm{bA}$ \\
\hline 9 & $4,5 \mathrm{dA}$ & $5,4 \mathrm{cA}$ & $4,2 \mathrm{bB}$ & $7,1 \mathrm{bA}$ & $4,7 \mathrm{cA}$ & $5,2 \mathrm{bA}$ & $0,75 \mathrm{bA}$ & $1,50 \mathrm{aA}$ \\
\hline 10 & $5,2 \mathrm{dA}$ & $6,6 \mathrm{bA}$ & $7,0 \mathrm{bA}$ & $7,0 \mathrm{bA}$ & $5,9 \mathrm{cB}$ & $6,3 \mathrm{bA}$ & $1,15 \mathrm{bA}$ & $1,23 \mathrm{bA}$ \\
\hline 11 & $5,0 \mathrm{~dB}$ & $7,2 \mathrm{aA}$ & $5,2 \mathrm{bA}$ & $6,4 \mathrm{bA}$ & $4,3 \mathrm{cA}$ & $5,8 \mathrm{bA}$ & $0,72 \mathrm{bA}$ & $0,65 \mathrm{bA}$ \\
\hline 12 & $5,6 \mathrm{~dB}$ & $7,6 \mathrm{aA}$ & $6,2 \mathrm{bA}$ & $8,2 \mathrm{bA}$ & $7,5 \mathrm{aA}$ & $8,1 \mathrm{aA}$ & $2,01 \mathrm{aA}$ & $2,13 \mathrm{aA}$ \\
\hline 13 & $6,2 \mathrm{dA}$ & $7,0 \mathrm{aA}$ & $6,8 \mathrm{bB}$ & $10,9 \mathrm{aA}$ & $6,2 \mathrm{bA}$ & $7,0 \mathrm{aA}$ & $0,50 \mathrm{bA}$ & $1,82 \mathrm{aB}$ \\
\hline 14 & $6,4 \mathrm{~dB}$ & $8,1 \mathrm{aA}$ & $8,7 \mathrm{aA}$ & $8,7 \mathrm{aA}$ & $7,4 \mathrm{aA}$ & $7,9 \mathrm{aA}$ & $0,90 \mathrm{bA}$ & $1,25 \mathrm{bA}$ \\
\hline 15 & $5,5 \mathrm{dA}$ & $5,8 \mathrm{bA}$ & $7,3 \mathrm{bA}$ & $9,4 \mathrm{aA}$ & $5,2 \mathrm{cA}$ & $6,0 \mathrm{bA}$ & $0,69 \mathrm{bB}$ & $1,99 \mathrm{aA}$ \\
\hline
\end{tabular}

Medias seguidas por la misma letra minúscula (en la columna) y mayúscula (en la línea) no difieren entre sí por la prueba de Scott y Knott al 5\% de probabilidad. I1 y I2 representan, respectivamente: $100 \%$ y $150 \%$ ET.

parte aérea, tal vez por el hecho de que son los sustratos con menores valores de densidad aparente (cuadro 3). La densidad en función de la granulometría y las partículas en los sustratos 1,2 y 3 pudo haber proporcionado mayor porosidad, favoreciendo un mayor almacenamiento de agua por el sustrato y su consecuente disponibilidad para la planta. La combinación de los sustratos Plantmax ${ }^{\circledR}$ y estiércol proporcionó ganancias significativas en el crecimiento y desarrollo de las plantas de T. heptaphylla.

Estas combinaciones de sustratos sirvieron para el desarrollo y crecimiento de las plantas, mejorando significativamente el crecimiento de las mismas.

No hubo diferencia significativa entre los niveles de irrigación utilizados para la altura de la parte aérea a los 135 y 150 DDS, para el diámetro de la base del tallo de la planta a los 75 y 120 DDS y para el número de hojas a los 135 y 150 DDS (cuadro 4), probablemente porque no tuvo buenas condiciones de drenaje, lo cual se manifestó en los niveles de crecimiento. Los análisis estadísticos indicaron la existencia de interacción sustrato e irrigación para la altura de la parte aérea a los 90 días después de la siembra (DDS) y para el número de hojas a los 75 días después de la siembra (DDS) (cuadro 5). De modo general se verifica que a pesar que hubo interacción sustrato-irrigación los valores medios fueron altos, dando a entender que tanto los sustratos como la irrigación no actuaron como se esperaba sobre el desarrollo de las plantas.

Los datos relativos a la materia seca de la raíz y parte aérea son presentados en el cuadro 6 . Se verificó que hubo diferencia significativa entre los sustratos estudiados, pues las plantas de los tratamientos 1,2 y 3 presentaron mayores valores para la materia seca de la raíz. Y para materia seca de la parte aérea los sustratos 2 y 3 fueron los que presentaron mayores valores medios. Estos sustratos propiciaron el mejor desarrollo de la raíz y de la parte aérea porque crearon un ambiente favorable. Este hecho probablemente se debe a la adecuada relación entre aire, agua y disponibilidad de nutrientes, ya que en aquellos sustratos que contenían compuesto de residuo urbano la liberación de nutrientes fue más lenta. 
Cuadro 6. Resultado del análisis de varianza para materia seca de la raíz y parte aérea después de 150 días de la siembra (DDS) en 15 tratamientos utilizados para la especie Tabebuia heptaphylla.

Result of analysis of variance: Dry matter of the acre ad seedling portion and dry matter of root system 150 days after sowing in 15 treatments used for the species Tabebuia heptaphylla.

\begin{tabular}{ccc}
\hline $\begin{array}{c}\text { Sustratos } \\
(\mathrm{S})\end{array}$ & $\begin{array}{c}\text { Materia seca de la } \\
\text { raíz }(\mathrm{g})\end{array}$ & $\begin{array}{c}\text { Materia seca de la } \\
\text { parte aérea }(\mathrm{g})\end{array}$ \\
\hline 1 & $0,67 \mathrm{a}$ & $0,67 \mathrm{c}$ \\
2 & $0,92 \mathrm{a}$ & $1,41 \mathrm{a}$ \\
3 & $0,42 \mathrm{a}$ & $1,74 \mathrm{a}$ \\
4 & $0,31 \mathrm{~b}$ & $0,62 \mathrm{c}$ \\
5 & $0,21 \mathrm{~b}$ & $0,77 \mathrm{c}$ \\
6 & $0,52 \mathrm{~b}$ & $0,79 \mathrm{c}$ \\
7 & $0,45 \mathrm{~b}$ & $0,37 \mathrm{c}$ \\
8 & $0,54 \mathrm{a}$ & $0,38 \mathrm{c}$ \\
9 & $0,50 \mathrm{~b}$ & $0,53 \mathrm{c}$ \\
10 & $0,41 \mathrm{~b}$ & $0,49 \mathrm{c}$ \\
11 & $0,46 \mathrm{~b}$ & $0,31 \mathrm{c}$ \\
12 & $0,38 \mathrm{~b}$ & $0,75 \mathrm{c}$ \\
13 & $0,53 \mathrm{~b}$ & $0,54 \mathrm{c}$ \\
14 & $0,48 \mathrm{~b}$ & $0,54 \mathrm{c}$ \\
15 & $0,44 \mathrm{~b}$ & $0,54 \mathrm{c}$ \\
\hline Valor $\mathrm{F}$ & $4,41 * *$ & $2,85 * *$ \\
\hline
\end{tabular}

Medias seguidas por la misma letra, en la columna no difieren entre sí al $5 \%$ de probabilidad por la prueba de Scott y Knott. $* * P<0,01$

Se pudo verificar que hubo interacción significativa sustrato-irrigación solamente para las relaciones altura de la parte aérea/diámetro de la base del tallo de la planta y materia seca de la parte aérea/materia seca de la raíz (cuadro 7). Los factores sustrato e irrigación actuaron conjuntamente sobre el desarrollo de las plantas para esas relaciones estudiadas.

\section{DISCUSIÓN}

El pH con valores 4,2, 6,2 y 6,9, para los tratamientos 1,2 y 3 , respectivamente (cuadro 3 ), en conjunto con la irrigación, además del probable efecto de la densidad de sustrato en la cantidad de agua disponible, pueden haber proporcionado mayor disponibilidad de nutrientes y desarrollo de las plantas de T. heptaphylla. Los valores de $\mathrm{pH}$ en $\mathrm{CaCl}_{2}$ superiores a 6,0 generan una acidez que es clasificada como muy baja (Raij 1991).

Se verificó que los mayores valores de $\mathrm{pH}$ (cuadro 3) ocurrieron en los sustratos cuyas composiciones contenían estiércol o compuesto de residuo urbano. El aumento de los valores de $\mathrm{pH}$ en suelos ácidos, cuando son sometidos a la aplicación de compuesto de residuo urbano, puede
Cuadro 7. Resultado del análisis de varianza para las relaciones altura/diámetro de la base del tallo (H/D), altura/materia seca de la parte aérea (H/MSPA), materia seca de la parte aérea/materia seca de la raíz (MSPA/MSR) e índice de calidad de Dickson (IDC) para plántulas de Tabebuia heptaphylla en respuesta a diferentes sustratos y niveles de irrigación (100\% y $150 \%$ ET) a los 150 DDS.

Result of analysis of variance: relation between plant height/ stem-base diameter, relation between plant height/dry matter of the acre ad seedling portion, total dry matter produced, the relation between plant height/stem diameter, dry matter of the acre ad seedling portion/dry matter of root system and Dickson quality index 150 days after sowing for seedlings Tabebuia heptaphylla in response to different substrates and levels of irrigation (100\% and $150 \%$ ET) to the 150 DDS.

\begin{tabular}{|c|c|c|c|c|c|}
\hline Sustratos (S) & $\mathrm{H} / \mathrm{D}$ & $\begin{array}{l}\text { MSR+ } \\
\text { MSPA }\end{array}$ & H/MSPA & $\begin{array}{l}\text { MSPA/ } \\
\text { MSR }\end{array}$ & IDC \\
\hline 1 & $6,45 \mathrm{~b}$ & $1,58 \mathrm{a}$ & $18,8 \mathrm{~b}$ & $1,18 \mathrm{c}$ & $0,21 \mathrm{a}$ \\
\hline 2 & $8,06 \mathrm{a}$ & $1,94 \mathrm{a}$ & $19,0 \mathrm{~b}$ & $1,12 \mathrm{c}$ & $0,21 \mathrm{a}$ \\
\hline 3 & $7,30 \mathrm{a}$ & $1,30 \mathrm{~b}$ & $33,4 \mathrm{a}$ & $0,92 \mathrm{c}$ & $0,16 \mathrm{~b}$ \\
\hline 4 & $7,77 \mathrm{a}$ & $1,05 \mathrm{~b}$ & $20,8 \mathrm{~b}$ & $2,42 \mathrm{a}$ & $0,11 \mathrm{~b}$ \\
\hline 5 & $6,79 \mathrm{~b}$ & $1,19 \mathrm{~b}$ & $17,2 \mathrm{~b}$ & $1,72 \mathrm{~b}$ & $0,14 \mathrm{~b}$ \\
\hline 6 & $6,49 \mathrm{~b}$ & $1,02 \mathrm{~b}$ & $25,4 \mathrm{a}$ & $1,17 \mathrm{c}$ & $0,13 \mathrm{~b}$ \\
\hline 7 & $6,11 \mathrm{c}$ & $1,02 \mathrm{~b}$ & $26,4 \mathrm{a}$ & $0,80 \mathrm{c}$ & $0,15 \mathrm{~b}$ \\
\hline 8 & $4,87 \mathrm{~d}$ & $0,84 \mathrm{~b}$ & $30,2 \mathrm{a}$ & $0,70 \mathrm{c}$ & $0,15 \mathrm{~b}$ \\
\hline 9 & $5,17 \mathrm{~d}$ & $0,99 \mathrm{~b}$ & $20,1 \mathrm{~b}$ & $1,17 \mathrm{c}$ & $0,16 \mathrm{~b}$ \\
\hline 10 & $5,95 \mathrm{c}$ & $0,94 \mathrm{~b}$ & $22,9 \mathrm{~b}$ & $1,05 \mathrm{c}$ & $0,14 \mathrm{~b}$ \\
\hline 11 & $5,81 \mathrm{c}$ & $0,78 \mathrm{~b}$ & $29,9 \mathrm{a}$ & $1,02 \mathrm{c}$ & $0,12 \mathrm{~b}$ \\
\hline 12 & $7,52 \mathrm{a}$ & $1,30 \mathrm{~b}$ & $18,4 \mathrm{~b}$ & $1,80 \mathrm{~b}$ & $0,14 \mathrm{~b}$ \\
\hline 13 & $6,63 \mathrm{~b}$ & $1,02 \mathrm{~b}$ & 30,6 a & $1,05 \mathrm{c}$ & $0,13 \mathrm{~b}$ \\
\hline 14 & $7,34 \mathrm{a}$ & $0,94 \mathrm{~b}$ & $32,3 \mathrm{a}$ & $1,22 \mathrm{c}$ & $0,11 \mathrm{~b}$ \\
\hline 15 & $5,36 \mathrm{~d}$ & $0,92 \mathrm{~b}$ & $22,4 \mathrm{~b}$ & $1,17 \mathrm{c}$ & $0,14 \mathrm{~b}$ \\
\hline Valor $\mathrm{F}$ & $12,33 * *$ & $3,77 * *$ & $1,54 \mathrm{NS}$ & $4,33 * *$ & $2,87 * *$ \\
\hline $\begin{array}{l}\text { Niveles de } \\
\text { Irrigación (I) }\end{array}$ & $\mathrm{H} / \mathrm{D}$ & $\begin{array}{l}\text { MSR+ } \\
\text { MSPA }\end{array}$ & H/MSPA & $\begin{array}{l}\text { MSPA/ } \\
\text { MSR }\end{array}$ & IDC \\
\hline $100 \%$ & $6,83 \mathrm{a}$ & $1,01 \mathrm{~b}$ & $27,7 \mathrm{a}$ & $1,12 \mathrm{a}$ & $1,14 \mathrm{a}$ \\
\hline $150 \%$ & $6,22 \mathrm{~b}$ & $1,25 \mathrm{a}$ & $21,2 \mathrm{~b}$ & $1,35 \mathrm{a}$ & $0,15 \mathrm{a}$ \\
\hline Valor F & $14,97 * *$ & $7,10 *$ & $8,71 * *$ & $4,36^{\mathrm{ns}}$ & 0,94 ns \\
\hline $\mathrm{S} \times \mathrm{I}$ & $2,24 * *$ & 0,73 ns & $1,65 \mathrm{~ns}$ & $2,09 *$ & 0,94 ns \\
\hline $\mathrm{CV} \%$ & 9,40 & 30,92 & 34,90 & 34,95 & 28,98 \\
\hline
\end{tabular}

Medias seguidas por la misma letra, en la columna no difieren entre sí

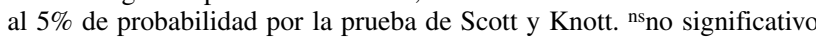
$(P>0,05) .{ }^{*} P<0,05$. $* * P<0,01$. CV: coeficiente de variación, $\mathrm{S} \times \mathrm{I}$ : interacción entre sustrato e irrigación.

ser atribuido a: la aplicación de humus alcalinos (Kiehl 1985), la producción de hidroxilo $\left(\mathrm{OH}^{-}\right)$, la acción del oxígeno de la solución del suelo que actúa como receptor de electrones provenientes de la oxidación microbiana del carbono (Mattiozzo-Prezotto 1992), y la capacidad de consumo de protones $\left(\mathrm{H}^{+}\right)$(Wong et al. 1998) y de $\mathrm{H}^{+}+\mathrm{Al}^{3+}$ por la carga orgánica del compuesto (Oliveira 2000); también, indirectamente, al aumento en los tenores de cationes cambiables del suelo $\mathrm{Ca}^{2+}, \mathrm{Mg}^{2+}, \mathrm{K}^{+}$y $\mathrm{Na}^{+}$ 
(Mansur et al. 1983). Tales mecanismos deben actuar simultáneamente para el aumento del $\mathrm{pH}$ en el suelo y parece ser que el principal de ellos es el cambio de $\mathrm{H}^{+}$ entre el sistema tampón del suelo y de la materia orgánica del compuesto (Wong et al. 1998, Oliveira 2000). Los antecedentes descritos permiten suponer que tales mecanismos también ocurren en los sustratos en cuya composición haya compuesto orgánico.

En el presente trabajo, este aumento del $\mathrm{pH}$ parece no haber favorecido el desarrollo de las plantas, limitando la disponibilidad de los nutrientes para las plantas, concordando con Fachini (2002), donde el pH del sustrato de compuesto de residuo urbano fue de 7,8, siendo 2,5 puntos mayor que el de sustrato de corteza de Pinus caribea. Este $\mathrm{pH}$ produce baja disponibilidad de nutrientes para las plantas, tornando al sustrato poco adecuado para su crecimiento. Según Handreck y Black (1999), el efecto del pH sobre la disponibilidad de nutrientes en un medio altamente orgánico es diferente comparado con los suelos naturales. De acuerdo con estos autores, el pH óptimo para tales medios debe estar en torno al 0,5, a una unidad abajo del recomendado para los suelos. Esto probablemente ocurrió en los sustratos 1, 2 y 3 del presente estudio.

Hubo variación entre los niveles de irrigación con las variables y los períodos evaluados (cuadro 4). Por lo tanto, para esta especie la necesidad de agua fue variable con el estado de desarrollo de la planta. En este sentido Lima (1993) observó que en la formación de plántulas de cítricos la necesidad de agua es variable, estando extremamente relacionada a los estados de desarrollo de la planta. Se puede verificar que las plantas de los tratamientos del nivel de irrigación $150 \%$ ET se destacaron en relación a las del nivel $100 \%$ ET. El mejor desempeño de las plantas en el nivel de irrigación $150 \%$ de ET concuerda con Loures et al. (1998), quienes evaluaron el cultivo de Lycopersicum esculentum Wild. en sustratos y en invernadero.

En las propiedades físicas del suelo los beneficios del compuesto orgánico, como reducción de la densidad aparente, mayor retención de agua y mayor estabilidad de agregados, son factores importantes para la mejoría de las condiciones de los suelos destinados a la agricultura, pudiendo todavía revertir las condiciones de los suelos pobres, tornándolos económicamente viables (Kiehl 1985).

Varias investigaciones demuestran que la incorporación de materia orgánica al suelo promueve mejorías en las propiedades físicas, repercutiendo favorablemente en la productividad agrícola (Berton et al. 1989, Galbiatti 1992, Nuvolari et al. 1996). El efecto físico causado por la materia orgánica en el suelo es muy importante para el crecimiento de los vegetales; según Henin et al. (1976), esta mejoría está relacionada, también, con el régimen de agua, pues mejorando la capacidad de infiltración se acelera el proceso dinámico del agua en el suelo. Su efecto en la mejoría de la estructura del suelo constituye un factor positivo para el crecimiento radicular. Esto fue evidenciado para T. heptaphylla, donde los sustratos 2 y 3 proporcionaron valores elevados para materia seca, siendo que éstos presentaron estiércol en su constitución. (Carvalho Filho et al. 2004) corrobora que el estiércol de bovino es el más usado y con buenos resultados en la producción de plantas de especies forestales como es constatado en la presente investigación. Por lo tanto, lo que puede haber influenciado en los menores valores de materia seca, al utilizar compuesto de residuo urbano en el sustrato, pudo haber sido el $\mathrm{pH}$ con valores elevados (cuadro 3). Con ello se confirmaría uno de los efectos de adición de compuestos orgánicos, la elevación del pH en el suelo, también observado por Hernando et al. (1989), Raij (1991) y Coker y Matthews (1983). De ese modo, el control del pH en la utilización de compuesto de residuo urbano puede ser un factor fundamental para posibilitar mejores resultados en la producción de plantas.

Entre las relaciones de los parámetros morfológicos usados para evaluar la calidad de plántulas de especies arbóreas (cuadro 7), la relación altura de la parte aérea combinada con el respectivo diámetro del cuello de la plántula (H/D) constituye uno de los más importantes atributos morfológicos para estimar el crecimiento de las plántulas después del establecimiento en el campo (Carneiro 1985). A través de los resultados, se observó que estos valores fueron favorables para el establecimiento de plántulas después del plantío. Para Pinus taeda L., Carneiro (1976) plantea que valores que varían de 5,4 a 8,1 son los ideales para la relación H/D. En el presente trabajo se pudo verificar que todos los valores están en este rango y presentaron menores valores los sustratos 8 , 9 y 15. Según Carneiro (1985), cuanto menor sea este valor, mayor será la capacidad de supervivencia y de establecimiento de las plantas.

El índice obtenido por la división de la altura de la parte aérea por la materia seca de la parte aérea puede ser de gran valor si es utilizado para predecir el potencial de supervivencia de las plántulas en el campo, a pesar de no ser comúnmente usado como un índice para evaluar el patrón de calidad de plantas en campo. Cuanto menor es este índice, más lignificada está la planta y mayor deberá ser la capacidad de supervivencia de ésta en el campo (Gomes 2001). Se puede verificar que hubo variación de esos valores en los sustratos estudiados, y que proporcionaron menores valores los sustratos $1,2,4,5,10,12$ y 15 , llevando a tener mayor expectativa de supervivencia para esos sustratos.

La relación entre materia seca de la parte aérea y la materia seca de la raíz es considerada como un índice eficiente y seguro para expresar la calidad de las plantas (Parviainen 1981). Se propuso que 2,0 sería la mejor relación entre estos atributos (Brissete y Barnett 1991). En el presente estudio hubo gran variación de esos valores (0,92 a 2,42), sin embargo presentó valor superior a 2,0 solamente el sustrato 4 .

Estableciendo como valor mínimo de IDC de 0,20, recomendado por Hunt (1990), se observa que las plántulas 
de T. heptaphylla apenas alcanzaron esos valores en los sustratos 1 y 2 , indicando que presentan calidad suficiente para ser plantadas en campo definitivo.

Sustratos que en su composición contienen mayor tenor de materia orgánica y también poseen elevada porosidad total, como en el caso del sustrato Plantmax ${ }^{\circledR}$, presentan buena capacidad de retención de agua y aireación, produciendo así plántulas con mejor crecimiento (Silva et al. 2000). Los principales efectos de los sustratos se manifiestan sobre las raíces, influyendo sobre el crecimiento de la parte aérea de la planta (Hartmann et al. 1990). Esa afirmación puede ser entendida al observar los resultados obtenidos en el presente estudio con la combinación de Plantmax ${ }^{\circledR}$ (cuadro 4) en los sustratos 2 y 3 que promovieron mayor valor de altura de la parte aérea y, consecuentemente, del diámetro de la base del tallo de la planta. Los sustratos 1, 2 y 3 proporcionaron los mayores valores en 90 a 150 DDS. Pio et al. (2005) señalan que el sustrato Plantmax ${ }^{\circledR}$ proporciona los mejores resultados en la producción de plántulas de Myrciaria jaboticaba (Vell.) O. Berg, promoviendo mayor tamaño de la parte aérea y del sistema radicular.

\section{REFERENCIAS}

Berton RS, OA Camargo, JMAS Valadares. 1989. Absorção de nutrientes pelo milho em resposta a adição do lodo de esgoto a cinco solos paulistas. Revista Brasileira de Ciência do Solo 13(1): 187-192.

Brissete JC, TD Barnett. 1991. Container seedlings. In Duryea ML eds. Forest Regeneration Manual. Dordrecht, The Netherlands. Kluwer Academic Publishers. p 117-141.

Broner I, RAP Law. 1991. Evaluation of modified atmometer for estimating reference ET. Irrigation Scientia.12(1): 21-26.

Caldeira MVW, MV Schumacher, N Tedesco. 2000. Crescimento de mudas de Acacia mearnsii em função de diferentes doses de vermicomposto. Scientia Florestalis, Piracicaba 57: 161-170.

Carneiro JGA. 1985. Produção e controle de qualidade de mudas florestais. Curitiba, Brasil. FUPEF. 451 p.

Carneiro JGA. 1976. Determinação do padrão de qualidade de mudas de Pinus taeda L. para plantio definitivo. Tesis Magíster en Agronomía. Curitiba, Brasil. Universidad Federal de Paraná. 70 p.

Carvalho Filho JLS, Arrigoni-Blank MF, Blank AF. 2004. Produção de mudas de Andira fraxinifolia Benth. em diferentes ambientes, recipientes e substratos. Ciência Agronômica 35(1): 61-67.

COELBA (Companhia de Eletricidade do Estado da Bahia, BR). 2002. Guía de Arborización Urbana. 56 p.

Coker EG, PJ Matthews. 1983. Metals in sewage sludge and their potential effects in agriculture. Water Science and Technology 1(15): 209-225.

Dickson A, A Leaf, JF Hosner. 1960. Quality appraisal of white spruce and white pine seedling stock in nurseries. Forestry Chronicle 36(1): 10-13.

Ettori LC, ACMF Siqueira, AS Sato, OR Campos. 1996. Variabilidade genética em populações de Ipê-roxo - Tabebuia heptaphylla (Vell.) Tol. - para conservação ex situ. Revista do Instituto Florestal 8(1): 61-70.
Fachini E. 2002. Diferentes níveis de irrigação e a utilização do composto de lixo orgânico na formação de mudas cítricas. Tesis de Maestria em Ciencias de Suelos. Jaboticabal, Brasil. Facultad de Ciencias Agrarias y Veterinarias, Universidad Estadual Paulista. 68 p.

Galbiatti JA. 1992. Efeito do uso contínuo de efluente de biodigestores sobre algumas características físicas do solo e o comportamento do milho Zea mays L. Tesis de Libre Docencia. Jaboticabal, Brasil. Facultad de Ciencias Agrarias y Veterinarias, Universidad Estadual Paulista. 212 p.

Gomes JM. 2001. Parâmetros morfológicos na avaliação da qualidade de mudas de Eucalyptus grandis produzidas em diferentes tamanhos de tubetes e de dosagens de N-P-K. Tese Doutorado em Ciência Florestal. Viçosa, Brasil. Universidade Federal de Viçosa. 166 p.

Gonçalves JLM, BV Raij, JC Gonçalves. 1997. Florestais. In Raij BV, H Cantarella, JA Quagio, AçMC Furlani eds. Recomendações de adubação e calagem para o Estado de São Paulo. Campinas, Brasil. Fundação IAC. p. 245-259.

Gonçalves JLM, F Poggiani. 1996. Substratos para produção de mudas florestais. In Congreso Latino Americano de Ciencia de Suelos. Piracicaba, Brasil. Sociedad Latinoamericana de Ciencia de Suelos CD-ROM.

Guerrini A, RM Trigueiro. 2004. Atributos físicos e químicos de substratos compostos por biossólidos. Revista Brasileira de Ciência do Solo, Viçosa 28(6): 1069-1076.

Handreck K, N Black. 1999. Growing media for ornamental plants and turf. Sydney, Australia. University of New South Wales Press. 448 p.

Hartmann HT, DE Kester, FT Davies. 1990. Plant propagation. New York, USA. Prentice Hall. 647 p.

Henin S, R Gras, G Monnier. 1976. Os solos agrícolas. Rio de Janeiro, Brasil. Forense Universitária. 327 p.

Hernando S, MC Lobo, A Polo. 1989. Effect of the application of a municipal refuse compost on the physical and chemical properties of a soil. Science of the Total Environment 1(81): $589-596$.

Hunt GA. 1990. Effect of stryroblock design and cooper treatament on morphology of conifer seedlings. In Taget Seedlings Symposium, Meeting of the Western Forest Nursery Associations. Roseburg, USA. Foresty Service. p. 218-222.

IBGE (Instituto Brasileiro de Geografia e Estatística, BR) 2005. Consultado 23 may. 2005. Disponible en ww.ibge.gov.br/ home/estatistica/populacao/condicaodevida/pnsb/lixocoletado/ defaultlixo.shtm.

Kiehl EJ. 1985. Fertilizantes orgânicos. São Paulo, Brasil. Agronômica Ceres. 492 p.

Köppen W. 1973. Das geographische System der Klimate. Berlin. Gebruder Borntraeger. 369 p.

Lima JEO. 1993. Produção de mudas na áfrica do Sul. São Paulo. Laranja 14(1): 127-136.

Longhi RA. 1995. Livro das árvores. Árvores e arvoretas do Sul. 2 ed. Porto Alegre, Brasil. L\&PM. 176 p.

Loures JL, PCR Fontes, MAN Sediyma, VWD Casali, AA Cardoso. 1998. Produção e teores de nutrientes no tomateiro em substrato contendo esterco de suíno. Horticultura Brasileira 16(1): 50-55.

Mattiozzo-Prezotto ME. 1992. Química Ambiental e Agronomia. In Dechen AR, AE Boareto e FC Verdade eds. Reunião 
Brasileira de Fertilidade do Solo e Nutrição de Plantas. Campinas, Brasil. Fundação Cargill. p. 157-78.

Nuvolari A, B Coraucci-Filho, PS Fadini, MB DaSilva. 1996. Aplicação de lodo de esgoto municipal no solo. Influência na capacidade de campo. In XI Congresso Nacional de Irrigação e Drenagem. Campinas, Brasil. ANAIS. p. 403-411.

Oliveira FC. 2000. Disposição de lodo de esgoto e composto de lixo urbano num Latossolo Vermelho Amarelo cultivado com cana-de-acúcar. Tesis de Doctorado en Agronomia. Piracicaba, Brasil. Escuela Superior de Agricultura "Luiz de Queiroz", Universidade de San Pablo. 207 p.

Parviainen JV. 1981. Qualidade e avaliação da semente de mudas florestais. In Seminário de Sementes e Viveiros Florestais. Curitiba, Brasil. FUPEF. 59-90 p.

Pio R, JD Ramos, TCA Gontijo, EP Carrijo, V Mendonça, EG Fabri, EA Chagas. 2005. Substratos na produção de mudas de Myrciaria jaboticaba. Agrociência 11(4): 393-396.

Raij BV. 1991. Fertilidade do solo e adubação. Piracicaba, Brasil. Ceres. 343 p.
San Martin E. 2000. Lixo urbano: um artigo de luxo no Brasil. São Paulo: Banas Ambiental 4: 26-31.

Schmitz JAK, PVD Souza, AN Kampf. 2002. Propriedades químicas e físicas de substratos de origem mineral e orgânica para o cultivo de mudas em recipientes. Ciência Rural Santa Maria 32(6): 937-944.

Scott AJ, MA Knott. 1974. Cluster analysis method for grouping means in the analysis of variance. Biometrics 30: 507-512.

Silva ACR, HS Fernandes, SR Martins. 2000. Produção de mudas de alface com vermicomposto em diferentes tipos de bandejas. Horticultura Brasileira 18(1): 512-513.

Tomé Júnior JB. 1997. Manual para interpretação de análise do solo. Guaíba, Brasil. Agropecuária. 247 p.

Wong MTF, S Nortcliff, RS Swift. 1998. Method for determining the acid ameliorating capacity of plant residue compost, urban waste compost, farmyard manure, and peat applied to tropical soils. Communications in Soil Science and Plant Analysis 29: 2927-2937. 